\title{
Protocolo de solicitação de ressonância magnética do joelho em pacientes idosos com suspeita de osteoartrose: Redução da solicitação de exames e o impacto na conduta e no diagnóstico*
}

\section{Protocol for the Request of Knee Magnetic Resonance Imaging in Elderly Patients with Suspected Osteoarthritis: Reduction in Test Requests and Impact on Management and Diagnosis}

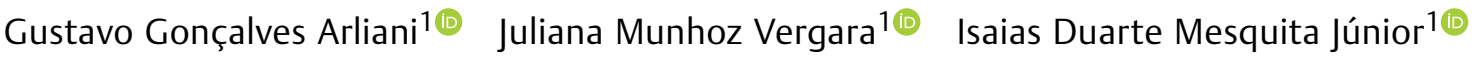 \\ Victor Otávio Oliveira ${ }^{1}$ Paulo Henrique Schmidt Lara10 Gabriel Ferraz Ferreira ${ }^{10}$ \\ ${ }^{1}$ Departamento de Ortopedia e Traumatologia, Instituto Prevent
Senior, São Paulo, SP, Brasil \\ Rev Bras Ortop 2022;57(3):409-414. \\ Endereço para correspondência Gabriel Ferraz Ferreira, MD, MSc, \\ Departamento de Ortopedia e Traumatologia, Instituto Prevent \\ Senior, Rua Cerro Corá, 585, Sala 605, Torre 1, São Paulo, SP, Brasil \\ (e-mail: gabriel.ferraz38@yahoo.com.br).
}

\section{Resumo \\ Palavras-chave \\ - osteoartrite do joelho \\ - imagem por ressonância magnética \\ - protocolos clínicos \\ Objetivo Desenvolver um protocolo, baseado em evidências, para guiar a solicitação de exames de ressonância magnética (RM) em pacientes idosos com suspeita de osteoartrose do joelho e avaliar a sua eficácia após implementação. \\ Métodos $\mathrm{O}$ protocolo institucional foi desenvolvido após revisão da literatura durante o primeiro semestre do ano de 2018. Definiu-se como grupo de controle os pacientes do primeiro semestre de 2018 , antes da aplicação/divulgação do protocolo institucional, e o grupo de estudo foi composto por pacientes atendidos no segundo semestre do mesmo ano após a padronização dos pedidos de RM para a suspeita de osteoartrose do joelho. \\ Resultados Nossa amostra contou com 826 pacientes submetidos a RM do joelho, com média de idade de 69,3 anos. Após a implementação do protocolo, houve um decréscimo das solicitações de RM e um aumento no número de solicitações de radiografias $(p<0,001)$. Após a implementação do protocolo, a RM alterou o diag- nóstico ou a conduta do médico em apenas $11,2 \%$ dos casos. \\ Conclusão Após a introdução do protocolo, encontrou-se uma redução de $47,5 \%$ no número de pedidos de RM do joelho, sendo que a maioria (89\%) dos pacientes não tiveram suas condutas ou diagnóstico alterados. Nível de evidência: estudo caso-controle (IIIB).}

Trabalho desenvolvido no Departamento de Ortopedia e Trauma-

tologia, Instituto Prevent Senior, São Paulo, Brasil.

recebido

15 de Junho de 2020

aceito

02 de Outubro de 2020

Publicado on-line

Março 31, 2021
DOI https://doi.org/

10.1055/s-0040-1722576.

ISSN 0102-3616.
(C) 2021. Sociedade Brasileira de Ortopedia e Traumatologia. All rights reserved.

This is an open access article published by Thieme under the terms of the Creative Commons Attribution-NonDerivative-NonCommercial-License, permitting copying and reproduction so long as the original work is given appropriate credit. Contents may not be used for commercial purposes, or adapted, remixed, transformed or built upon. (https://creativecommons.org/ licenses/by-nc-nd/4.0/)

Thieme Revinter Publicações Ltda., Rua do Matoso 170, Rio de Janeiro, RJ, CEP 20270-135, Brazil 


Abstract
Keywords
- osteoarthritis, knee
- magnetic resonance
imaging
- clinical protocols

Objective To develop an evidence-based protocol to guide magnetic resonance imaging (MRI) requests in elderly patients with suspected knee osteoarthrosis and to evaluate its effectiveness after implementation.

Methods The institutional protocol was developed after reviewing the literature during the first semester of 2018. The control group was defined as patients cared for in the first semester of 2018 , before the implementation/dissemination of the institutional protocol, and the study group was composed by patients cared for during the second semester of 2018 after the standardization of MRI requests for suspected knee osteoarthrosis.

Results Our sample included 826 patients undergoing knee MRI, with a mean age of 69.3 years. Protocol implementation decreased MRI requests and increased radiograph requests $(p<0.001)$. After the implementation of the protocol, the MRI changed the diagnosis or treatment in only $11.2 \%$ of the cases.

Conclusion Protocol implementation resulted in a $47.5 \%$ reduction in the number of requests for knee MRI, with most $(89 \%)$ patients with alteration in diagnosis or treatment. Level of evidence: case-control study (IIIB).

\section{Introdução}

A osteoartrose (OA) é uma doença degenerativa crônica considerada um problema de saúde pública. Isto porque é a doença articular mais prevalente no mundo, e a causa isolada mais comum de incapacidade em indivíduos maiores de 18 anos. A articulação mais acometida pela $\mathrm{OA}$ é a do joelho, sendo muito prevalente em pacientes com idade superior a 50 anos, e atualmente afeta algo em torno de 250 milhões de pessoas no planeta. ${ }^{1}$

No Brasil, com o rápido envelhecimento da população associado ao aumento epidêmico da obesidade, deve ocorrer um crescimento exponencial de pacientes com suspeita e diagnóstico de OA do joelho nos próximos anos. ${ }^{2}$

O diagnóstico da OA normalmente é estabelecido por meio da anamnese, e dos exames clínico e radiográfico, sendo a radiografia o método de imagem mais utilizado, por ser barato, amplamente disponível e validado, e por facilitar a classificação da gravidade da doença. A utilização de outros exames subsidiários, como a ressonância magnética (RM), pode ser importante em situações específicas.

A RM é especialmente útil para se confirmar a suspeita do diagnóstico quando a avaliação clínica e os achados das radiografias são divergentes ou duvidosos, uma vez que, além de ser um método não invasivo de obtenção de imagens multiplanares, apresenta alta definição, sensibilidade e especificidade. $^{3}$

A escolha do exame mais indicado e adequado faz com que a doença possa ser diagnosticada mais rapidamente, aumentando a probabilidade de sucesso no tratamento, além disso, evitando custos desnecessários ao sistema de saúde. ${ }^{4}$

No entanto, tratar de custos na área da saúde é complexo, uma vez que se discute o bem estar e a vida humana. ${ }^{5}$ Desta forma, a discussão não deve centrar-se somente nos custos, como também, no esforço para que o serviço seja realizado com a máxima eficiência e qualidade. ${ }^{6}$
Assim sendo, desenvolver um protocolo, baseado na literatura médica e de fácil utilização, que guie a solicitação de exames de RM em pacientes idosos com suspeita de OA do joelho é de suma importância, pois permite não só melhorar a abordagem terapêutica ao paciente, como também provoca a diminuição de pedidos desnecessários de RM, com melhor distribuição e uso dos recursos de saúde disponíveis. ${ }^{7}$

O objetivo deste estudo é desenvolver um protocolo, baseado em evidências, para guiar a solicitação de exames de RM em pacientes idosos com suspeita de OA do joelho e avaliar a sua eficácia após a implementação.

\section{Materiais e Métodos}

O estudo contou com a avaliação de 22.654 consultas de pacientes ambulatoriais da ortopedia do Hospital Sancta Maggiore, em São Paulo, atendidos por médicos ortopedistas especialistas em joelho durante o período de $1^{\circ}$ de janeiro a 31 de dezembro de 2018. Após a aplicação dos critérios de inclusão e exclusão, totalizaram-se 826 pacientes com suspeita de OA de joelho que foram submetidos ao exame de RM.

\section{Protocolo Institucional}

A estratégia metodológica consistiu na pesquisa nos bancos de dados do PubMed e SciELO para identificar estudos sobre diagnóstico de $\mathrm{OA}$ e dor no joelho publicados nos cinco anos anteriores ao início da pesquisa. A busca foi direcionada para artigos escritos em português e inglês, com acesso total, público e eletrônico. A combinação das palavras-chave utilizada na busca foi: knee osteoarthritis, knee pain, diagnosis, MRI e magnetic resonance.

As publicações de interesse foram selecionadas inicialmente a partir da leitura do título e do resumo. Também foram analisadas as publicações relevantes citadas nos artigos escolhidos. 
Editoriais e cartas ao editor não foram incluídos. Após a seleção final dos artigos e a leitura integral dos mesmos, propôs-se o protocolo a seguir, atualizado e específico para a solicitação de exame de RM do joelho em pacientes idosos com diagnóstico ou suspeita diagnóstica de OA.

Critérios para a solicitação de RM do joelho em pacientes maiores de 60 anos com dor crônica ( $>6$ meses) no joelho e suspeita/diagnóstico de OA do joelho:

- Realização prévia de radiografias nas incidências anteroposterior (AP) e de perfil (P) do joelho com carga;

- Histórico de trauma/entorse agudo do joelho;

- Sinais e Ssntomas de bloqueio articular;

- Testes meniscais positivos, sem presença de dor difusa no joelho;

- Dor aguda/súbita no joelho, sem trauma (suspeita de OA);

- Dor desproporcional aos achados presentes nas radiografias;

- Suspeita de fratura por insuficiência em pacientes com osteoporose; e

- Suspeita de tumoração no joelho.

\section{Seleção dos Pacientes}

Os critérios de inclusão foram: 1) idade acima de 60 anos; 2) suspeita ou diagnóstico de OA do joelho; e 3) atendimento no ambulatório de ortopedia da instituição no ano de 2018. Já os critérios de exclusão foram: 1) osteoartrose secundária; 2) pacientes com doenças inflamatórias sistêmicas; 3 ) pedidos de RM com finalidades diversas, como laudo, relatório e fins trabalhistas; e 4) prontuário com informações insuficientes.

\section{Aplicação e Avaliação do Protocolo}

0 protocolo institucional foi desenvolvido durante o primeiro semestre de 2018, e, após a sua conclusão e revisão, foi massivamente divulgado nos diversos canais de contato com os médicos ortopedistas especialistas em joelho da instituição (e-mail, intranet, Whatsapp) durante o segundo semestre do mesmo ano.

Desse modo, definiu-se como grupo de controle os pacientes antendidos no primeiro semestre de 2018, antes da aplicação/divulgação do protocolo institucional, e o grupo de estudo foi composto por pacientes atendidos no segundo semestre após a padronização dos pedidos de RM por suspeita de OA do joelho.

\section{Análise Clínica}

Os fatores incluídos na análise clínica foram: idade, gênero, lateralidade, número de RMs solicitadas em cada semestre, número de consultas em cada grupo, existência de solicitação prévia (nos doze meses anteriores) de radiografias do joelho e alteração da conduta, ou o diagnóstico do paciente após análise do exame de RM.

\section{Análise Estatística}

A análise estatística foi realizada usando-se o pacote stats do programa R ( $R$ Foundation for Statistical Computing, Viena, Áustria). ${ }^{8}$ As variáveis nominais foram descritas pela sua medida de proporção, e as contínuas, pela média e desvio padrão. Realizou-se o teste de Shapiro ${ }^{9}$ para avaliar a distribuição das variáveis contínuas em cada grupo, e aplicou-se o

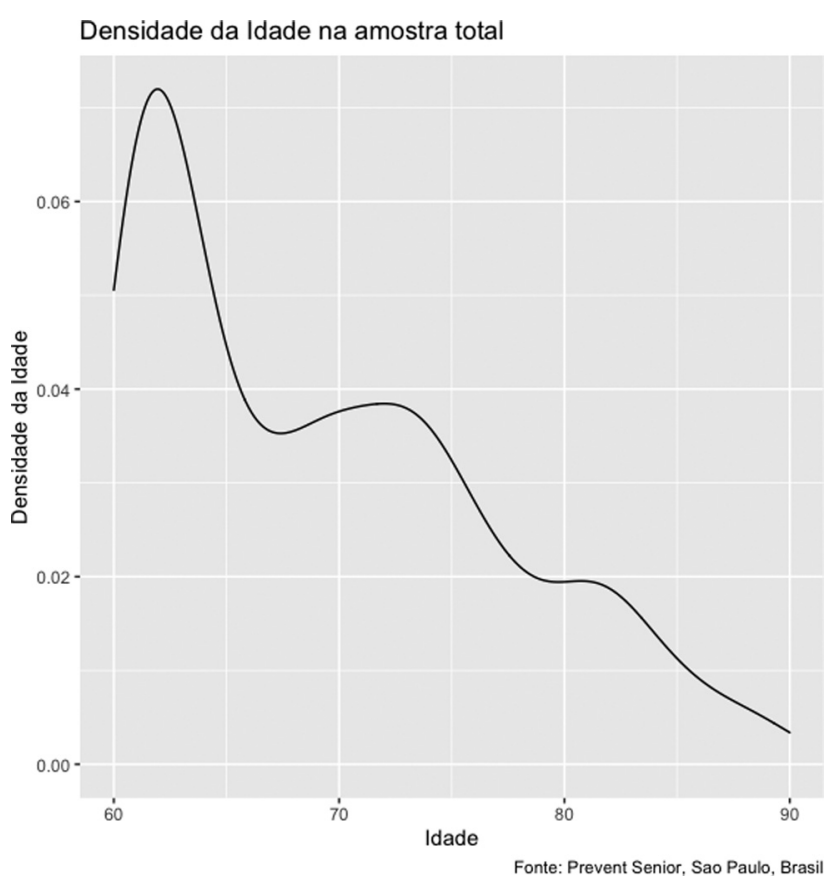

Fig. 1 Densidade da idade ao longo da amostra total.

teste $t$ de Student para as paramétricas, e o teste U de MannWhitney para as não paramétricas. Realizou-se o teste do qui-quadrado de Pearson ${ }^{10}$ para a comparação das variáveis categóricas entre os grupos. A significância estatística foi estabelecida com valores de $p \leq 0.05$.

\section{Resultados}

A amostra total foi de 22.654 consultas médicas com ortopedistas especialistas em joelho, sendo 10.869 consultas no grupo de controle (primeiro semestre de 2018) e 11.785 no grupo de estudo (segundo semestre de 2018), e demonstrou uma similaridade entre o número de consultas realizadas nos dois períodos. A idade média da amostra foi de 69,3 anos. Podese observar a densidade da distribuição da idade na - Figura 1.

As características epidemiológicas dos grupos podem ser observadas na - Tabela 1.

Houve uma redução de $47.5 \%$ no número de pedidos de RM pelos médicos ortopedistas especialistas em joelho após a divulgação do protocolo institucional. Essa redução variou de 559 (67,7\%) pedidos no primeiro semestre de 2018 para 267 (32,3\%) solicitações deste exame no segundo semestre, como se pode observar na - Figura 2.

Quando analisamos o número de RMs em cada grupo relacionado ao número de consultas, chegamos ao valor de duas RMs para cada cem consultas após a instauração do protocolo. Já no grupo de controle, ou seja, antes do protocolo, encontrou-se a relação de cinco pedidos de RM para cada cem consultas, totalizando uma redução aproximada de três exames a cada cem consultas.

Em relação às radiografias solicitadas previamente à RM, os pedidos aumentaram após a implementação do protocolo, com uma elevação de quase $96 \%$. A porcentagem de radiografias prévias foi de $23,9 \%$ no grupo de controle, e de $47,1 \%$ 
412 Protocolo de solicitação de ressonância magnética do joelho Arliani et al.

Tabela 1 Características dos pacientes incluídos no estudo

\begin{tabular}{|c|c|c|c|}
\hline Característica & Antes do protocolo & Após o protocolo & Valor de $p$ \\
\hline Idade & $69,3(7,6)^{*}$ & $69,4(7,9)^{*}$ & 0,62 \\
\hline Sexo & $\begin{array}{l}\text { Feminino }=73,7 \% \\
\text { Masculino }=26,3 \%\end{array}$ & $\begin{array}{l}\text { Feminino }=69,1 \% \\
\text { Masculino }=30,9 \%\end{array}$ & 0,34 \\
\hline Lateralidade & $\begin{array}{l}\text { Direito }=50,5 \% \\
\text { Esquerdo }=49,5 \%\end{array}$ & $\begin{array}{l}\text { Direito }=44,7 \% \\
\text { Esquerdo }=55,3 \%\end{array}$ & 0,22 \\
\hline $\begin{array}{l}\text { A radiografia foi solicitada previamente } \\
\text { à ressonância magnética? }\end{array}$ & $\begin{array}{l}\text { Sim }=23,9 \% \\
\text { Não }=76,1 \%\end{array}$ & $\begin{array}{l}\text { Sim }=47,1 \% \\
\text { Não }=52,9 \%\end{array}$ & $p<0,001$ \\
\hline $\begin{array}{l}\text { O exame de ressonância magnética alterou } \\
\text { a conduta ou o diagnóstico? }\end{array}$ & $\begin{array}{l}\text { Sim }=13,8 \% \\
\text { Não }=86,2 \%\end{array}$ & $\begin{array}{l}\text { Sim }=11,2 \% \\
\text { Não }=88,8 \%\end{array}$ & 0,30 \\
\hline
\end{tabular}

Fonte: Dados do Departamento de Ortopedia e Traumatologia, Instituto Prevent Senior, São Paulo, Brasil.

Nota: *Média e desvio padrão.

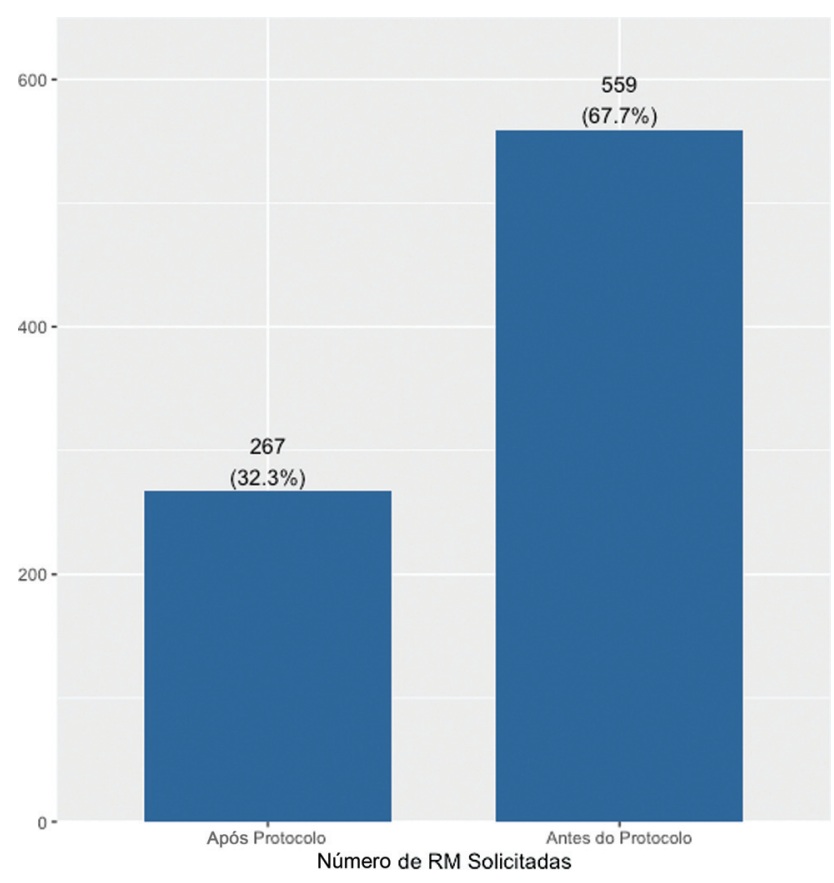

Fig. 2 Número de ressonâncias magnéticas (RMs) solicitadas nos grupos de estudo e de controle.

do grupo de estudo (- Figura 3), sendo considerada a diferença estatisticamente significativa $(p<0.001)$.

Após análise do exame de ressonância magnética, a conduta ou diagnóstico sofreram uma alteração de $11.2 \%$ nos pacientes no grupo de estudo, e em $13.8 \%$ no grupo de controle, sem diferença significativa entre os grupos $(p=0.30)$.

\section{Discussão}

Os principais resultados deste estudo foram o desenvolvimento de um protocolo institucional, baseado na literatura vigente, para solicitação de exames de RM do joelho em pacientes idosos com suspeita de OA do joelho e posterior demonstração da sua eficácia após implementação na equipe de ortopedistas especialistas em joelho da instituição.

No presente estudo, observamos uma redução de 52\% no número de pedidos de RM do joelho após a implementação do protocolo institucional. Isto representou uma diminuição
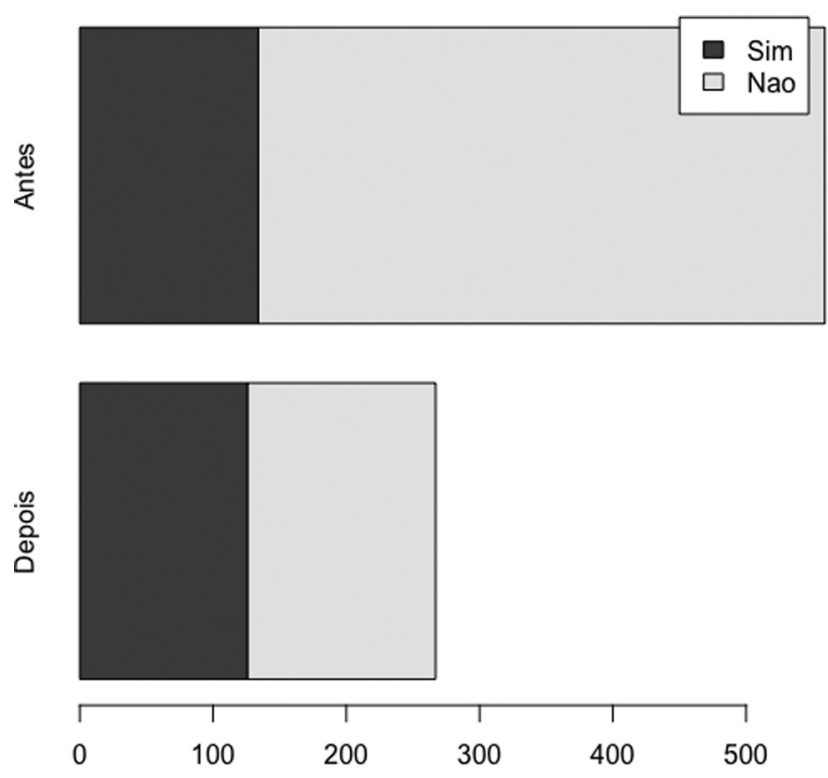

Número de radiografias solicitadas previamente a RM

Fig. 3 Número de radiografias solicitadas previamente ao pedido de RM nos grupos de estudo e de controle.

de 292 exames no segundo semestre do ano, ou seja, aproximadamente 49 RMs de joelho a menos por mês. Esssas vagas puderam ser ocupadas com outros pedidos de RM mais urgentes e essenciais, diminuindo o tempo e a fila de espera para a realização desses exames. Spence et al. ${ }^{7}$ encontraram resultados semelhantes com redução de $71 \%$ de pedidos inapropriados de RM do joelho em pacientes com OA depois da implementação do protocolo. A queda proporcionou a liberação de 45 vagas por mês para realização de exames mais urgentes e necessários.

Já Kandiah et al. ${ }^{11}$ demonstraram uma queda de $21 \%$ nas requisições de RMs do joelho, ombro e quadril após o desenvolvimento e a aplicação de protocolo para solicitação destes exames em pacientes maiores de 55 anos de idade. Os resultados encontrados foram inferiores aos observados em outros estudos; no entanto, estes achados podem ter ocorrido devido à análise conjunta de pedidos de exames de diversas articulações, como as do ombro e do quadril. 
As diferenças encontradas em relação a outros estudos prévios também podem ser relacionadas aos médicos solicitantes dos exames, visto que outros autores ${ }^{5,12}$ demonstraram diferenças nos padrões de solicitação de exames entre ortopedistas gerais, ortopedistas especialistas, e médicos de atenção primária. A implantação do protocolo desenvolvido neste estudo foi realizada somente em médicos especialistas em joelho.

Outro achado importante deste trabalho foi o aumento de $96 \%$ no número de pedidos de radiografias do joelho após divulgação do protocolo institucional. A mudança proporcionou uma queda de $76 \%$ para $52 \%$ no número de pacientes com suspeita diagnóstica de OA que realizavam RM do joelho sem ao menos ter realizado radiografias anteriormente. Apesar da redução observada, os números ainda demonstram uma baixa adesão do protocolo vigente, visto que um dos critérios presentes não foi respeitado nessas solicitações.

Gonzalez et al. ${ }^{13}$ também observaram baixa aderência ao protocolo (57\%), por parte de médicos da atenção primária, num estudo com pacientes com dor não aguda do joelho e média de idade dos participantes de 53 anos. Glover et al., ${ }^{14}$ num estudo que avaliou pedidos de RM de extremidades no pronto-socorro, demonstraram que a razão mais comum para pedidos inapropriados de exames de RM foi a não realização de radiografias prévias. Parent et al., ${ }^{15}$ em um estudo que avaliou idosos, observaram que somente $38 \%$ dos pacientes submetidos a RM do joelho tinham sido submetidos a radiografias do joelho nos 24 meses anteriores.

Observamos neste estudo que aproximadamente $86 \%$ dos pacientes não tiveram suas condutas alteradas após a análise do resultado do exame de RM solicitado pelo médico. Outro estudo $^{13}$ semelhante demonstrou que aproximadamente $20 \%$ dos pacientes tiveram suas condutas clínicas alteradas pelos resultados dos exames de RM do joelho. E Lehnert et al. ${ }^{16}$ demonstraram que $76 \%$ dos exames gerais de RM solicitados por médicos generalistas apresentavam resultados normais, e o tratamento dos pacientes não sofreu mudanças.

O desenvolvimento de protocolos institucionais orientando as práticas médicas é fundamental para padronização e tomada acertada de decisões. Além disso, estes guias proporcionam um melhor uso dos finitos e escassos recursos financeiros da área da saúde. Parker et al. ${ }^{17}$ projetaram custo de 2 bilhões de dólares em 2020 somente com pedidos de RM musculo-esqueléticas para beneficiários do Medicare nos Estados Unidos. Desta forma, o surgimento e aplicação de protocolos nesta área é vital para um melhor controle dos gastos, evitando desperdícios.

Apesar de a classificação da OA do joelho ter sido descrita por Kellgren-Lawrence, ${ }^{18}$ ela permanece atual para o diagnóstico e a conduta em casos de OA do joelho, mesmo com algumas divergências entre os achados radiológicos e clínicos. Já a RM é um exame mais sensível e específico do que a radiografia, podendo ser utilizado em algumas situações, haja vista que o exame não altera o diagnóstico e a classificação radiográfica.
Este estudo apresenta algumas limitações. Por ter sido realizado em uma única instituição, com equipe própria de ortopedia e facilidade de comunicação interna, fica difícil generalizar os achados encontrados para outros serviços. Segundo, o protocolo foi implementado somente para médicos especialistas de joelho, não tendo, desta maneira, comparação com médicos generalistas e da atenção primária. Também não foram avaliados os motivos para não adesão e seguimento, por parte dos médicos, do protocolo desenvolvido.

\section{Conclusão}

Após o desenvolvimento e a implementação de um protocolo institucional para a solicitação de exames de RM do joelho em pacientes idosos com suspeita de OA, observamos uma redução 47.5\% no número de pedidos de RM do joelho, um aumento de $96 \%$ no número de pedidos iniciais de radiografias do joelho, e que a maioria (89\%) dos pacientes não tiveram suas condutas alteradas após a análise do resultado do exame de RM solicitado pelos médicos.

\section{Suporte Financeiro}

Não houve suporte financeiro de fontes públicas, comerciais, ou sem fins lucrativos.

\section{Conflito de Interesses}

Os autores declaram não haver conflito de interesses.

\section{Referências}

1 Vos T, Flaxman AD, Naghavi M, et al. Years lived with disability (YLDs) for 1160 sequelae of 289 diseases and injuries 1990-2010: a systematic analysis for the Global Burden of Disease Study 2010. Lancet 2012;380(9859):2163-2196

2 Heidari B. Knee osteoarthritis prevalence, risk factors, pathogenesis and features: Part I. Caspian J Intern Med 2011;2(02): 205-212

3 Rodrigues MB, Camanho GL. MRI Evaluation of Knee Cartilage. Rev Bras Ortop 2010;45(04):340-346

4 Bettmann MA. The ACR Appropriateness Criteria: view from the committee chair. J Am Coll Radiol 2006;3(07):510-512

5 Sherman SL, Gulbrandsen TR, Lewis HA, et al. Overuse of Magnetic Resonance Imaging in the Diagnosis and Treatment of Moderate to Severe Osteoarthritis. Iowa Orthop J 2018;38(01):33-37

6 Bautista AB, Burgos A, Nickel BJ, Yoon JJ, Tilara AA, Amorosa JKAmerican College of Radiology Appropriateness. Do clinicians use the American College of Radiology Appropriateness criteria in the management of their patients? AJR Am J Roentgenol 2009;192 (06):1581-1585

7 Spence SC, McAlister W, Reed B, et al. A Multispecialty Collaboration to Reduce Unnecessary Imaging for Knee Osteoarthritis. J Am Coll Radiol 2016;13(11):1343-1346

8 R: A language and environment for statistical computing [computer program]. Vienna, Austria: R Foundation for Statistical Computing; 2014

9 Shapiro SS. WILK MB. An analysis of variance test for normality. Biometrika 1965;52(3-4):591-611

10 Pearson KX. On the criterion that a given system of deviations from the probable in the case of a correlated system of variables is such that it can be reasonably supposed to have arisen from random sampling. Lond Edinb Dublin Philos Mag J Sci 1900;50 (302):157-175 


\section{Protocolo de solicitação de ressonância magnética do joelho Arliani et al.}

11 Kandiah JW, Chan VWY, Luo J, Dong F, Nugent JP, Forster BB. Reducing the Volume of Low-Value Outpatient MRI Joint Examinations in Patients $\geq 55$ Years of Age. Can Assoc Radiol J 2020;71 (01):83-91

12 Roberts TT, Singer N, Hushmendy S, et al. MRI for the evaluation of knee pain: comparison of ordering practices of primary care physicians and orthopaedic surgeons. J Bone Joint Surg Am 2015;97(09):709-714

13 Gonzalez FM, Kerchberger JM, Robertson DD, et al. Knee MRI Primary Care Ordering Practices for Nontraumatic Knee Pain: Compliance With ACR Appropriateness Criteria and Its Effect on Clinical Management. J Am Coll Radiol 2019;16(03): 289-294

14 Glover M, Gottumukkala RV, Sanchez Y, et al. Appropriateness of Extremity Magnetic Resonance Imaging Examinations in an
Academic Emergency Department Observation Unit. West J Emerg Med 2018;19(03):467-473

15 Parent ME, Vézina F, Carrier N, Masetto A. Indications for and clinical procedures resulting from magnetic resonance imaging of the knee in older patients: Are we choosing wisely? Can Fam Physician 2018;64(03):e126-e132

16 Lehnert BE, Bree RL. Analysis of appropriateness of outpatient CT and MRI referred from primary care clinics at an academic medical center: how critical is the need for improved decision support? J Am Coll Radiol 2010;7(03):192-197

17 Parker L, Nazarian LN, Carrino JA, et al. Musculoskeletal imaging: medicare use, costs, and potential for cost substitution. J Am Coll Radiol 2008;5(03):182-188

18 Kellgren JH, Lawrence JS. Radiological assessment of osteo-arthrosis. Ann Rheum Dis 1957;16(04):494-502 